\title{
A stochastic model for forecast consumption in master scheduling
}

\author{
P.J. Weeda \\ Department of Mechanical Engineering, University of Twente, P.O. Box 217, 7500 AE Enschede, The Netherlands
}

\begin{abstract}
This paper describes a stochastic model for the reduction of the initial forecast in the Master Schedule (MS) of an MRP system during progress of time by the acceptance of customer orders.

Results are given for the expectation and variance of the number of yet unknown deliveries as a function of futurity within the planning horizon. The expectation will be a measure for the forecast. The result for the variance is a measure for the forecasting error and may act as a basis for the amount of safety stock or available to promise, to project in the MS.
\end{abstract}

\section{Introduction}

The phenomenon of consuming the forccast by actual customer orders during the process of time in the Master Schedule (MS) is well known and described in many textbooks on production and inventory management. However, its relationship with the probability distribution of the lead time desired by customer orders is usually neglected. Moreover, no consideration is given with respect to the relation between the required amount of safety stock or available to promise as a function of futurity in the MS and the customer desired lead time distribution. In order to develop the model proposed here, the following assumptions are made:

(1) The customer desired lead times are independent and identically distributed random variables with a given distribution function.

(2) The deliveries of customer orders can be modelled by a renewal process, generating the time intervals between successive deliveries.
(3) The desired lead times of the delivered orders are independent of the renewal process of the deliveries.

The organization of this paper is as follows. In Section 2 the stochastic model is developed. Results for the expectation and variance of the number of yet unknown future deliveries are given in terms of the customer desired lead time distribution and the mean and the variance of the renewal process of the deliveries. Some numerical results will be presented in Section 3.

\section{The stochastic model}

In order to construct the model, it is assumed that a customer with a desired lead time $L$ will put his order at $L$ time units in advance of its desired due date. A customer order, which has to be delivered at a future time $t$, is already known at present $(t=0)$, if its desired lead time $L$ satisfies 
$L \geqslant t$. A customer order, which has to be delivered at a future time $t$ is still unknown at present, if its desired lead time $L$ satisfies $L<t$.

Let the distribution function of the customer desired lead time be denoted by $H(t)=P\{L<t\}$. Then $H(t)$ specifies the fraction of customer orders to be delivered at future time $t$ which are still unknown at $t=0$, while $1-H(t)$ specifies the fraction of customer orders to be delivered at time $t$, which are known at $t=0$.

A second assumption is concerned with the time intervals between successive deliveries of customer orders. It is assumed that these intervals $X_{1}$, $X_{2}, \ldots$ are mutually independent and identically distributed random variables with distribution function $F(x)-P\{X<x\}$. In other words, the delivery process can be represented by a renewal process $\{X\}$, with distribution function $F(x)$.

A third assumption is the independence between the customer desired lead time of a delivery and the renewal process of the deliveries $\{X\}$. This assumption implies that the customer desired lead times of successive deliveries should also be independent and identically distributed random variables with distribution function $H(t)$.

Further renewal processes are considered, which are imbedded in the basic renewal process of the deliveries $\{X\}$. Let the random variable $Y_{t}$ denote the time interval between two successive deliveries with a desired lead time $L<t$. Its distribution function is denoted by $G_{t}(y)=P\left\{Y_{t}<y\right\}$. A time interval $Y_{t}$ consists of a geometrically distributed number of intervals of process $\{X\}$. Hence, the distribution function $G_{t}(y)$ is given by

$$
G_{t}(y)=H(t) \sum_{n=1}^{x}[1-H(t)]^{n-1} F_{n}(y)
$$

where $F_{n}(y)$ represents the $n$-fold convolution of the distribution function of the renewal process $\{X\}$.

Let the random variable $A_{t}$ denote the number of deliveries per time unit at a future time $t$, which are unknown at time $t=0$, i.e. the orders with a desired lead time $L<t$. Then according to a result from renewal theory (cf. [1]) its expectation $E\left(A_{t}\right)$ is related to the expected time interval between deliveries $E\left(Y_{t}\right)$ by means of

$$
E\left(A_{t}\right)=E\left(Y_{t}\right)^{-1} \text {, }
$$

where $E\left(Y_{t}\right)$ can be obtained from

$$
\begin{aligned}
E\left(Y_{t}\right) & =H(t) \sum_{n=1}^{x}[1-H(t)]^{n \cdot 1} n E(X) \\
& =E(X) H(t)^{-1} .
\end{aligned}
$$

Hence, the expected number of deliveries per time unit with desired lead time $L<t$ is given by

$E\left(A_{t}\right)=H(t) E(X)^{-1}$,

where $E(X)^{-1}$ is the expected number of deliveries per time unit. Note that the result explains the role of the customer desired lead time distribution $H(t)$ in the illustrations of forecast consumption in the literature, for example in [2] and [3] in order to clarify the differences between make-to-stock, assemble-to-order and make-to-order environments. $E(X)^{-1}$ represents the maximum forecast, while the time dependency during the planning horizon (i.e. the futurity) is obtained by multiplication with the customer desired lead time distribution function $H(t)$.

We next turn to the expression for the variance in the number of deliveries per time unit with desired lead time $L<t$. The outcome will be related to the forecast error and can be used to establish the desired size of the safety stock as a function of futurity $t$ in the planning horizon of the MS.

In order to obtain an expression for $\operatorname{Var}\left(Y_{t}\right)$, we note that $Y_{t}$ consists of a random sum of random variables. Using a well-known result in this respect (cf. [4]), $\operatorname{Var}\left(Y_{t}\right)$ is given by

$\operatorname{Var}\left(Y_{t}\right)=E(N) \operatorname{Var}(X)+\operatorname{Var}(N) E(X)^{2}$,

where $N$ is the geometrically distributed random variable representing the number of intervals of process $\{X\}$ in $Y_{1}$. According to a result from renewal theory (cf. [1]), $\operatorname{Var}\left(A_{t}\right)$ is related to $\operatorname{Var}\left(Y_{t}\right)$ by means of

$\operatorname{Var}\left(A_{t}\right)=\operatorname{Var}\left(Y_{t}\right) E\left(Y_{t}\right)^{-3}$

Eqs. (5) and (6) imply the following result for the variance in the number of deliveries per time unit with desired lead time $L<t$ or equivalently the variance of the yet unknown number of deliveries per time unit as a function of the futurity $t$ in the 
planning horizon:

$$
\begin{aligned}
\operatorname{Var}\left(A_{t}\right)= & {\left[\operatorname{Var}(X) H(t)^{2}+H(t)\{1-H(t)\}\right.} \\
& \left.\times E(X)^{2}\right] / E(X)^{3} .
\end{aligned}
$$

By means of this result, the amount of safety stock to keep in the MS as a function of the futurity $t$ can be established in the usual way by multiplying the square root of $\operatorname{Var}\left(A_{t}\right)$ by a safety factor.

Furthermore, since only $\operatorname{Var}(X)$ and $E(X)$ appear in expression (7), $\operatorname{Var}\left(A_{t}\right)$ may be expressed in $E\left(A_{t}\right), H(t)$ and the coefficient of variation $C(X)$ of the time intervals of renewal process $\{X\}$. This results in

$\operatorname{Var}\left(A_{t}\right)=E\left(A_{t}\right)\left[1-H(t)\left\{1-C(X)^{2}\right\}\right]$

or equivalently

$\operatorname{Var}\left(A_{t}\right)=\left[H(t)\{1-H(t)\}+H(t)^{2} C(X)^{2}\right] / E(X)$.

Denoting $E\left(A_{t}\right), \operatorname{Var}\left(A_{t}\right)$ for $t=0$ and $t \rightarrow \infty$, respectively, by $E\left(A_{0}\right), \operatorname{Var}\left(A_{0}\right)$ and $E\left(A_{\infty}\right), \operatorname{Var}\left(A_{\infty}\right)$, the following expression for these quantities are easily verified using (4) and (9) and assuming $H(0)=0$ and $H(t) \rightarrow 1$ for $t \rightarrow \infty:$

$E\left(A_{0}\right)=0, \quad \operatorname{Var}\left(A_{0}\right)=0$

and

$E\left(A_{\infty}\right)=E(X)^{1}, \quad \operatorname{Var}\left(A_{\infty}\right)=C(X)^{2} E(X)^{-1}$.

A quite surprising result is obtained if $\operatorname{Var}\left(A_{t}\right)$ is investigated on extrema. Investigating the firstorder derivative yields one and only one extremum at $t=t^{*}$, satisfying

$H\left(t^{*}\right)=\left[2\left(1-C(X)^{2}\right)\right]^{-1}$, for $C(X)<1$.

Substituting (12) in (9) yields

$$
\begin{aligned}
\operatorname{Var}\left(A_{\imath}^{*}\right)=\left[4\left(1-C(X)^{2}\right]^{-1} E(X)^{-1},\right. \\
\\
\text { for } C(X)<1,
\end{aligned}
$$

where $\operatorname{Var}\left(A_{\imath}^{*}\right)$ satisfies

$\operatorname{Var}\left(A_{0}\right)<\operatorname{Var}\left(A_{t}^{*}\right) \geqslant \operatorname{Var}\left(A_{\infty}\right)$

proving a unique maximum at $t=t^{*}$ for $C(X)<1$, where $t^{*}$ is the unique root of Eq. (12). Note that the results for $C(X)<1$ are surprising, since one would cxpect $\operatorname{Var}\left(A_{t}\right)$ to be a monotonically increasing function of the futurity $t$ in the planning horizon, bounded by $\operatorname{Var}\left(A_{\infty}\right)$, given by (11).

If the classes of Erlang- and Gamma-distributions are considered for the renewal intervals between successive deliveries, it is easily verified that

$C(X)^{2}=1 / n$, for $n \geqslant 1$

implying a unique maximum for

$H\left(t^{*}\right)=n / 2(n-1)$, for $n>1$.

For $C(X)=1$ (exponential distribution), (4) and (8) imply

$\operatorname{Var}\left(A_{t}\right)=E\left(A_{t}\right)=H(t) E(X)^{-1}$,

which assumes its maximum value $E(X)^{-1}$ for $t \rightarrow \infty$. Note that for $n=2$, (16) implies also $H\left(t^{*}\right)=1$, implying a maximum equal to $E(X)^{-1}$ at infinity for $n=2$ and explaining the $\geqslant$ sign in (14).

\section{Numerical examples}

In this section three numerical examples are presented. The customer desired lead time distribution is given by an Erlang-2 distribution function

$H(t)=1-\mathrm{e}^{-\alpha t}(1+\alpha t)$,

with $\alpha=0.5$. The delivery process of the customer orders is modelled, respectively, by an exponential, an Erlang-2 and an Erlang- 3 distribution. The expected number of deliveries per time unit is given by $E(X)^{-1}=10$ in all three cases.

Results are given for the expectation, the variance and the standard deviation of the random variable $A_{t}$, i.e. the number of deliveries per time unit in future time periods $t=1(1) 6$ and for $t \rightarrow \infty$ in the planning horizon of the MS, which are still unknown at present $(t=0)$. As explained in Section 2, this quantity is in fact the number of deliveries per time unit at time $t$ of orders with a desired lead time $L<t$, which are evidently not yet arrived at $t=0$. The expectation of $A_{t}$ acts as a forecast of the yet unknown number of deliveries. The standard deviation of $A_{t}$ may be considered as 
a measure of the forecast error, thus establishing a basis for a safety quantity as a function of future time in the MS. In Tables $1-3$ the results are presented.

The equality of expectation and variance in Table 1 is clear from relation (8) with $C(X)=1$. The variance and standard deviation of $A_{t}$ are larger then those for Erlang-2-distributed delivery intervals in Table 2 . In Table 3 the variance and standard deviation of $A_{t}$ on the short term are

\section{Table 1}

Results for an Erlang-2-distributed customer desircd lead time and exponentially distributed delivery intervals

\begin{tabular}{llllllll}
\hline$I$ & 1 & 2 & 3 & 4 & 5 & 6 & $x$. \\
$E\left(A_{t}\right)$ & 0.89 & 2.64 & 4.42 & 5.95 & 7.13 & 8 & 10 \\
$\operatorname{Var}\left(A_{t}\right)$ & 0.89 & 2.64 & 4.42 & 5.95 & 7.13 & 8 & 10 \\
$\sigma\left(A_{t}\right)$ & 0.94 & 1.61 & 2.10 & 2.44 & 2.67 & 2.83 & 3.16 \\
\hline
\end{tabular}

Table 2

Results for an Erlang-2-distributed customer desired lead time and Erlang-2-distributed delivery intervals

\begin{tabular}{llllllll}
\hline$t$ & 1 & 2 & 3 & 4 & 5 & 6 & $x_{-}$ \\
$E\left(A_{1}\right)$ & 0.89 & 2.64 & 4.42 & 5.95 & 7.13 & 8 & 10 \\
$\operatorname{Var}\left(A_{t}\right)$ & 0.88 & 2.28 & 3.44 & 4.18 & 4.59 & 4.80 & 5 \\
$\sigma\left(A_{t}\right)$ & 0.94 & 1.51 & 1.85 & 2.05 & 2.14 & 2.14 & 2.24 \\
\hline
\end{tabular}

Table 3

Results for an Erlang-2-distributed customer desired lead time and Erlang-3-distributed delivery intervals

\begin{tabular}{llllllll}
\hline$t$ & 1 & 2 & 3 & 4 & 5 & 6 & $x$ \\
$E\left(A_{t}\right)$ & 0.89 & 2.64 & 4.42 & 5.95 & 7.13 & 8 & 10 \\
$\operatorname{Var}\left(A_{t}\right)$ & 0.86 & 2.47 & 3.86 & 4.66 & 4.96 & 4.93 & 3.33 \\
$\sigma\left(A_{t}\right)$ & 0.93 & 1.59 & 1.96 & 2.16 & 2.23 & 2.24 & 1.82 \\
\hline
\end{tabular}

larger than those for Erlang-2-distributed delivery intervals, while in the long run the opposite is true. However, they are smaller than those for exponentially distributed delivery intervals. In Table 3 the existence of a maximum in the variance derived in Section 2 is confirmed computationally.

\section{Conclusions}

A stochastic model for forecast consumption has been developed, which shows the analytical relationship between the fraction of yet unknown deliveries as function of the futurity within the planning horizon and the customer desired lead time distribution. Also analytical results for the mean and the variance of the number of yet unknown deliveries as function of the futurity in the planning horizon are obtained. The result on the mean is in agreement with what should be expected. An unexpected outcome has been the fact that the variance in the yet unknown deliveries attains a unique maximum at a finite value of the futurity in the planning horizon. The results provide a basis for the determination of the necessary amount of safety stock or available to promise at a future time $t$ in the MS of an MRP system.

\section{References}

[1] Cox, D.R., 1967. Renewal Theory. Methuen, London.

[2] Fogarty, D.W., Blackstone, J.II. Jr. and Hoffmann, T.R., 1991. Production and Inventory Management, 2nd edn., South-Western Publishing, Cincinati, $\mathrm{OH}$.

[3] Vollmann, I.E., Berry, W.E. and Whybark, D.C.. 1988. Manufacturing Planning and Control Systems, 2nd edn., lrwin, Homewood. IL 60430.

[4] Feller, W., 1966. An Introduction to Probability Theory and its Applications, Vol. II. Wiley, New York. 